Las familias como copias.

Técnicas de Reproducción Humana Asistida (TRHA) y desigualdades reproductivas

María Cecilia Johnson

Con X (N. $\left.{ }^{\circ} 6\right)$, e034, 2020

ISSN 2469-0333 | https://doi.org/10.24215/24690333e034

http://perio.unlp.edu.ar/ojs/index.php/conequis

FPyCS | Universidad Nacional de La Plata

La Plata | Buenos Aires | Argentina

\title{
Las familias como copias
}

\section{Técnicas de Reproducción Humana Asistida (TRHA) y desigualdades reproductivas}

\section{The Families as Copies: \\ Assisted Reproductive Techniques (ARTs) and Reproductive Inequalities}

\section{María Cecilia Johnson \\ cecilia.johnson@unc.edu.ar \\ https://orcid.org/0000-0002-5102-2504}

Consejo Nacional de Investigaciones Científicas y Técnicas (CONICET)

Centro de Investigaciones y Estudios sobre Cultura y Sociedad (CIECS)

Universidad Nacional de Córdoba | Argentina

\section{Resumen}

A través de diversos relatos biográficos de usuarias de Técnicas de Reproducción Humana Asistida (TRHA) en la Argentina, se analizan un conjunto de intersecciones sociales que en el campo familiar expresan jerarquías, entendidas como parte de la estratificación reproductiva. Partiendo de relatos de mujeres usuarias de TRHA, se analizan las desigualdades que las identifican como copias frente a un original familiar heterosexual, conyugal y bioconectado y que se expresan en reconocimientos o en estigmas para sus familias.

\section{Palabras clave | estratificación reproductiva, heteronormatividad,} reproducción asistida

\begin{abstract}
Through different biographical narratives of users of Assisted Reproduction Techniques (ARTs) in Argentina, the article analyzes the social intersections that in the family field express hierarchies, understood as the stratification of reproduction. From the user's narratives, these stratifications will be taked as copies related to a heterosexual, marital, and bio-connected family, taken as the original that express recognitions and stigma to their families.
\end{abstract}

Keywords | reproductive stratification, heteronormativity, assisted reproduction 


\title{
Las familias como copias
}

\author{
Técnicas de Reproducción Humana Asistida (TRHA) \\ y desigualdades reproductivas
}

Por María Cecilia Johnson

Como señala Charis Thompson (2005), las Técnicas de Reproducción Humana Asistida (TRHA) «Son estructuradas alrededor de una estricta escenografía conyugal, heterosexual, orientada al consumo y nuclear-familiar. Cuando los tratamientos son exitosos les permiten a las mujeres a reinscribirse en dicha lógica» (p. 56). ${ }^{1}$ En línea con este razonamiento, las TRHA no solo producen bebés sino también madres / padres (Thompson, 2005), por lo que no es posible pensar estas técnicas sin pensar la construcción de sentidos sobre las familias que nacen producto de las mismas (Luna, 2001).

La asunción de que la familia como organización social se basa en una naturaleza biológica es una noción que las TRHA han contribuido a disputar en el espacio público. En la Argentina, este conjunto de transformaciones sobre el parentesco ha sido posible en un escenario político de creciente reconocimiento de derechos sexuales y reproductivos para una población históricamente excluida por apartarse de la matriz heteronormativa, situación que no fue ajena a disputas políticas dado que para algunos sectores supone poner en jaque a la familia natural (Peñas Defago, Moran Faundes \& Vaggione, 2018).

El reconocimiento de un grupo social como familia no ha sido democráticamente distribuido, sino que ha estado sujeto a un conjunto de intersecciones que en el campo familiar expresan desigualdades y formas de estratificación reproductiva (Colen, 1995; Rapp, 1999). Esta noción supone la identificación de una organización 
social que jerarquiza la salud reproductiva, la fecundidad y la crianza, y en cuyo marco algunas formas de maternidad consideradas normales son recompensadas frente a otras experiencias no normativas que son dejadas en el margen de lo no reconocido, patologizadas o relegadas a lo ilegal (Preciado, 2019; Rapp, 2001).

En el presente trabajo se propone explorar estas transformaciones sobre la familia y el parentesco desde los relatos biográficos de mujeres usuarias de TRHA, para analizar la manera en la que estas intersecciones se traducen en desigualdades. Para considerar tales jerarquías, se adopta la propuesta teórica performativa de Judith Butler (2002) y el planteo de Marla Brettschneider (2006), y se aborda la estratificación reproductiva a partir de considerar que algunas formas de reproducción y de parentesco no normativas constituyen copias frente a un original familiar nunca logrado.

Este planteo permite arribar a aquello que en los relatos de las usuarias de TRHA aparece como tensiones en sus experiencias reproductivas y familiares frente a un original familiar heteronormativo, conyugal y bioconectado. ${ }^{2}$ Algunas de las dimensiones que emergen en los relatos se vinculan a la conexión biológico genética como modelo hegemónico, a la heteronormatividad y a la conyugalidad, en tanto elementos que demarcan mayor o menor cercanía a este original familiar. La adopción también es retomada en esta sección como una experiencia que para algunas usuarias supone reflexionar sobre la pérdida de la consanguinidad en la construcción del parentesco y que, en ocasiones, es comparada con el uso de gametos donados, lo que muestra algunas conexiones y jerarquías que las usuarias asignan a estas prácticas.

Comprender las jerarquías y las desigualdades presentes en los relatos de usuarias de TRHA contribuirá a develar algunas lógicas de poder que dan cuenta de las resistencias de las personas usuarias de las instituciones y permitirá arribar a la pregunta por la transformación de lo familiar a partir de las TRHA.

\section{Materiales y métodos}

Desde el paradigma interpretativo de las ciencias sociales, y mediante el análisis de los relatos biográficos como subgénero de los relatos de vida (Conde, 1994), en este trabajo se busca comprender los sentidos que las usuarias de las TRHA 
le otorgan a las diversas formas de conformación de lo familiar, a partir de su propia experiencia y en su búsqueda por maternar.

En el marco de una investigación doctoral ${ }^{3}$ centrada en las experiencias biográficas de usuarias de las tecnologías de TRHA en la ciudad de Córdoba, Argentina, se trabajó con un diseño muestra de veintiún entrevistas a mujeres cis de diferentes edades, orientación sexual o erótica y estado conyugal. ${ }^{4}$ Las usuarias entrevistadas han realizado diferentes tipos de tratamientos de acuerdo a las clasificaciones de la biomedicina ${ }^{5}$ y fueron contactadas a partir de informantes clave y mediante el método de bola de nieve.

Asimismo, el presente trabajo da cuenta de un proceso de codificación de las entrevistas mediante un análisis cualitativo, que en varios casos relevaron como tópicos centrales la construcción de la familia y su tensión con el modelo hegemónico que supone la matriz heteronormativa y de conexión biológica. A partir de este criterio, en este artículo se retoman los relatos de nueve entrevistadas que, al momento de la entrevista, presentaban las siguientes situaciones: dos de ellas se encontraban en una relación sexo-afectiva con una mujer cis, dos habían realizado el tratamiento desde la monoparentalidad por elección (Jociles Rubio \& Rivas Rivas, 2016) y cinco se encontraban en una relación sexo-afectiva con un varón.

La muestra contempla diversas trayectorias y resultados con el tratamiento, tal es el caso de dos entrevistadas de este grupo que abandonaron el tratamiento con las TRHA y consiguieron adoptar. ${ }^{6}$ Cabe agregar, por último, que este trabajo se centra en el método biográfico interpretativo, por lo que no se pretende establecer un muestreo representativo sino arribar a los sentidos sobre la familia que se plantean desde la reflexividad de las propias usuarias en su vinculación con la temporalidad y con el contexto social (Sautu, Boniolo, Dalle \& Elbert, 2005).

\section{La reproducción estratificada: experiencias de usuarias de TRHA en la Argentina}

A partir de la última década, diversas estructuras familiares han cobrado mayor visibilidad y legitimidad en la escena social argentina, a partir de los reconocimientos jurídicos y sobre una diversidad de áreas que involucran derechos sexuales, reproductivos y de familia que antes eran estigmatizadas o invisibilizadas. 
Aquellos arreglos familiares que se diferencian del imaginario social construido en relación con la llamada familia heterosexual -privados, hasta entonces, de las cartas necesarias para lograr este reconocimiento- han sido los que lograron transformaciones con inevitables implicancias políticas, dado que no solo se disputa una representación o una idea de lo familiar, sino un principio fundamental que históricamente ha estructurado las relaciones sociales y que ha tenido consecuencias en el orden social y jurídico (Vaggione, 2008). Entendiendo que el orden legal tiene efectos concretos en las prácticas, las actitudes y las identidades sexuales que se consideran legítimas, así como en las que se consideran prohibidas, invisibilizadas y criminalizadas (Vaggione, 2012), es que las transformaciones en el derecho no son ajenas a las disputas en el campo social.

Ante la hegemonía vigente de una sociedad con claros rasgos heteronormativos, los movimientos feministas y de la diversidad sexual, o LGBT, centran sus esfuerzos en desinstitucionalizar el sistema de poder, de privilegios y de derechos que ubica al no-heterosexual en un lugar de opresión y de marginación (Vaggione, 2012), apelando a diferentes formas de reconocimiento (Vespucci, 2014). En el orden jurídico, en las últimas décadas los Estados latinoamericanos comenzaron a reconocer derechos de ciudadanía a las parejas de la diversidad sexual.

En la Argentina, ejemplos de ello son la Ley 1.004 de Unión Civil en la Ciudad Autónoma de Buenos Aires (2002), la Ley 26.618 de Matrimonio Civil (conocida como Ley de Matrimonio Igualitario) (2010) y la Ley 26.743 de Identidad de Género (2012) que ha sido un hito en el reconocimiento de derechos sexuales en el país. Del mismo modo, la Ley 26.862 de Reproducción Medicamente Asistida (2013) constituye, al menos en términos legales, un dispositivo médico tecnológico que reconoce -sin discriminar por orientación sexual y/o situación conyugal- el acceso a una amplia franja de la población y la cobertura dentro del Programa Médico Obligatorio $(\mathrm{PMO})^{7}$ y de la Salud Pública.

Del mismo modo, la reforma del Código Civil (2015) propuso algunas novedades vinculadas a la noción de voluntad y al énfasis en la elección de los sujetos sobre sus construcciones familiares por sobre los sentidos biologicistas de familia, que en el orden jurídico argentino fueron incorporadas bajo las formas de filiación reconocidas. Como refiere Marisa Herrera (2014), la especificidad de estas técnicas reside en que «hacen posible la disociación entre el elemento biológico, el genético y el volitivo, cobrando este último primacía» (p. 28). 
Estas trasformaciones en el campo jurídico, sin embargo, no se traducen de manera automática en las matrices culturales y en los imaginarios sobre lo familiar: no se puede desconocer que la norma simbólica del parentesco se ha construido sobre la base de cierto imaginario hegemónico de lo familiar heterosexual, conyugal, biológico y matrimonial. Como plantea Pierre Bourdieu (1997), el imaginario de lo familiar se internaliza y se hace cuerpo a lo largo de nuestra trayectoria de vida como habitus, y forma parte de nuestra construcción de mundo y de familia, por lo que podríamos pensar que pese a las transformaciones jurídicas este imaginario heteronormativo persiste en forma de habitus.

Desde una mirada performativa, Butler (2002) señala que el parentesco es una especie de hacer que no refleja una estructura anterior. No obstante, persiste la idea de una estructura de relaciones hipostasiada como la heterosexualidad, que en nuestra cultura ha estructurado y fundamentado el parentesco mismo. Sin embargo, este orden simbólico no es estático, sino que quienes se encuentran fuera del privilegio heterosexual disputan la apropiación de estos términos de dominación, resignificando los términos que consuman la exclusión (Butler, 2002), lo que puede llevarnos a pensar en una posibilidad de transformación no exenta de tensiones.

Siguiendo a Brettschneider (2006), se entiende que la adopción, el uso de gametas donadas y la construcción de familias no heteronormativas constituyen prácticas que tensionan a la biología y a la genética en la legitimación de los lazos familiares, así como a la conyugalidad y a la heterosexualidad como requisitos para la maternidad. Como refiere la autora, retomando el planteo de Butler (2002), la adopción como vía para conformar una familia suele ser tratada como una mímica, frente a las TRHA que se acercan más al llamado modelo natural.

[...] debemos ver que la familia «natural» es tan construida como la familia adoptiva "construida». Encontraremos que la situación no es tan simple como considerar que la familia biológica es dada, mientras que la familia adoptiva se hace; que la familia biológica es natural, mientras que la familia adoptiva está construida; o que la bio-familia es real u original, mientras que la adoptiva es una copia o espejo. En contraste, mostraré que, bajo escrutinio, el marco «las familias adoptivas son para la cultura lo que las bio-familias para la naturaleza» no se sostiene (Brettschneider, 2006, p. 81). ${ }^{8}$ 
Si nos ubicamos en el campo de la reproducción asistida, específicamente desde las trayectorias de las mujeres usuarias, existe una lógica que atraviesa la mayoría de los relatos vinculada con la búsqueda de una maternidad lo más cercana posible a la llamada familia original, entendida en nuestra cultura como la deseable: nuclear, heterosexual, biparental y genéticamente conectada. Esto es así porque las TRHA, como dispositivo tecnológico, se ofrecen a las personas usuarias desde la promesa de la reproducción biológica o genética.

En este sentido, el parentesco como proceso cultural implica, como refiere Butler (2002), cierta inteligibilidad; es decir, para que una familia pueda ser leída como tal se espera que se cumplan ciertas características. Si bien se advierte una creciente multiplicidad de sentidos a la hora de construir la familia -en cuyo marco el parentesco no solo está basado en lo biológico, en la sexualidad o en la heterosexualidad- cuando se quieren naturalizar la sexualidad y la reproducción lo que se pone en juego es la producción misma del sujeto, es decir, «quien será una persona socialmente reconocida como tal» (Butler, 2002, p. 16).

Más allá de estos aspectos, que dan cuenta de diversas formas de reproducción de la norma hegemónica del parentesco, un aspecto que Marilyn Strathern (1995) reconoce como una ruptura en el parentesco hegemónico es la diversificación de los papeles sociales: «Donantes, padres, madres, gestantes, dan cuenta de un desmembramiento de los papeles sociales» (p. 306), lo que podría poner en evidencia el carácter cultural de estas posiciones. Sin embargo, en la mayoría de los casos, esto no produce ningún tipo de ruptura cultural porque, como la autora, la tecnología en lugar de generar rupturas produce la sustitución de aquellos aspectos que se consideran significativos para el parentesco; en definitiva, la tecnología reproductiva aparece como sustituta de la relación sexual.

Se proponen a continuación un conjunto de categorías que de forma analítica buscan recuperar algunas dimensiones que aparecen intersecadas en los relatos de usuarias y que dan cuenta de un conjunto de jerarquías y de estratificaciones sobre las familias que se reproducen y se cuestionan en las propias experiencias. Sus relatos también evidencian procesos de creciente reflexividad que operan en las usuarias revelando la plasticidad y la maleabilidad de sentidos de lo biológico en el parentesco (Hayden, 1995), así como en la diversidad de arreglos familiares que, en la actualidad, las tecnologías reproductivas permiten asistir (Strathern, 1992). 
El desglose de categorías permite recuperar algunas nociones que aparecen, particularmente, cuando las usuarias reflexionan sobre la conformación de las propias familias y sobre los desafíos que encuentran frente a los obstáculos reproductivos. De esta manera, en cada apartado se identifican aspectos que refieren a rupturas de lo familiar: la heteronormatividad del modelo original de familia, en los casos de usuarias lesbianas o en una relación sexo-afectiva con otra mujer; la conyugalidad, en las experiencias de las madres solteras por elección; y la pérdida de consanguinidad, cuando se piensa la ovodonación y la adopción en paralelo. Todas estas dimensiones dan cuenta de una tensión no resuelta, que trasciende pero que al mismo tiempo atraviesa las experiencias particulares de las mujeres usuarias, así como de formas posibles de pensar lo familiar y los propios vínculos.

\section{El original heteronormativo}

Más allá de los avances sociales en materia de derechos de diversidad sexual y de las trayectorias de mujeres usuarias, la familia heterosexual aparece como modelo natural mediante el cual son pensadas otras relaciones de cuidado, estigmatizadas como malas copias que deben adaptarse a un sistema heteronormativo.

En la Argentina, la heteronormatividad presente en el reconocimiento de los vínculos familiares se muestra, durante la última década, en las luchas por el reconocimiento jurídico de otras formas familiares que no estuvieron exentas de tensiones (Sgró Ruata, 2012) y demuestran que el papel del Estado es central en la construcción y en la sedimentación de ciertos modelos legítimos de familia. Asimismo, estas luchas tienen un impacto en las subjetividades de quienes se encuentran por fuera de estos reconocimientos (Libson, 2012). Es por ello que esta dimensión aparece en los relatos de las usuarias vinculada a un conjunto de tensiones sociales en su impacto subjetivo y biográfico.

Así relataba Celina quien, consciente de su derecho a ser madre, no era ajena a las críticas que reciben las familias homoparentales, donde la heteronorma se traduce por momentos en forma de «culpa» y donde el imaginario de lo familiar hegemónico se encuentra internalizado: 
En algún momento, sí, me sentía con mucha culpa, por cómo fui criada. Mi papá me dijo: «Haciendo esto, vos le negás a ella un papá» / «Pero es que ella nunca tuvo un papá, fue un donante». Y bueno, yo a ella cuando la tengo en mis brazos le digo: «Vos estuviste congeladita en una heladera y mamá te deseó mucho, [...] lo fuimos a buscar al doctor y él nos ayudó». Siempre le voy contando como un cuentito y cuando ella vaya creciendo se lo voy a ir contando con más detalle (Celina, 43 años).

Al mismo tiempo que relata su culpa inicial, Celina reconoce que otras transformaciones sociales y políticas posibilitaron cierto resquebrajamiento de esta heteronormatividad, tanto a nivel personal como social. «Hubo muchos cambios, creo, a través del matrimonio igualitario. Creo que ahí hubo un gran cambio y como que un sector de la sociedad nos dejó de ver como enfermos» (Celina, 43 años).

Como señala Celina, la familia que no se ajusta a la norma heterosexual sufre el estigma de la patologización social de la personalidad, el género y el parentesco (Rapp, 2001). Esta patologización supone que ciertas formas de reproducción son problemáticas y estigmatizadas respecto a la norma, como sucede con el parentesco gay y lésbico. Según Rapp (2001), las tecnologías de la biomedicina también forman parte de las maneras en las que se patologiza la reproducción. Sin embargo, el relato de Celina también da cuenta de la politización de la propia experiencia, contracara de la estigmatización que, como señalan algunos/as autores/as, posibilita la conformación de nuevas identidades políticas (Rapp, 2001; Rose, 2007).

De hecho, en el proceso de su reflexión, Celina refiere que el cambio se produce en la dimensión cultural, esto es, que la naturalidad de la familia se relaciona con la aceptación y la recepción de la sociedad, y no con un carácter intrínseco de las familias heterosexuales.

Creo que todas las culpas y todas las cosas se fueron de nosotras cuando llegó [nombre de su hija]. Como que nos dimos cuenta que todo es cultural y que a pesar del tratamiento que hicimos, cuando nosotros se lo contamos a las personas, si las personas lo escuchan de manera natural lo toman naturalmente (Celina, 43 años). 
Si bien, como indica Strathern (1995) sobre la cultura euro-americana, en la crianza se tienden a aceptar diversos papeles sociales entendidos como verdaderos, sustitutos o adoptivos, la búsqueda, sin embargo, continúa siendo binaria y excluyente a la hora de identificar quiénes son madre o padre.

Los relatos de parejas lesbianas o de madres solteras por elección (Jociles Rubio \& Villamil Pérez, 2012), a diferencia de las parejas heterosexuales que han utilizado gametos donados, ponen en descubierto la no correlación entre sexualidad-procreación y permiten construir nuevas categorías y formas de vinculación y de identidad social. Esto, nuevamente, es claro en el proceso de reconocimiento de derechos, como le sucedió a Celina cuando quiso solicitar licencia por maternidad sin ser ella la gestante y la ley le quería reconocer su licencia bajo la categoría de padre, ${ }^{9}$ utilizando el formato legal heteronormativo como forma de discriminación.

La cuestión es que yo también tenía que presentar en mi hospital los papeles. Ya había hablado antes con la jefa de recursos humanos y le había contado sobre la técnica que íbamos a llevar a cabo. Ella me dijo: «Mirá, nunca le hemos dado la licencia por maternidad porque nunca se nos presentó un caso así, pero vamos a ver qué se puede hacer. Mientras tanto, vas a tener que tener la licencia como papá, que son quince días nada más» / «Pero es injusto, porque yo soy mamá, no soy papá. ¿Cómo voy a tener esa licencia?» / «Bueno, sí, pero hay que hacer esto». Pero yo no tenía la partida de nacimiento, no la tenía con mi nombre... (Celina, 43 años).

Estas formas de discriminación, como de disputa por el reconocimiento social, dan cuenta de la ruptura que la diversificación de los papeles sociales familiares genera en una sociedad heteronormativa. El conjunto de obstáculos que se les presentaron a Celina y a su pareja para la inscripción de su hija en el registro civil de Córdoba constituye otro ejemplo de la heteronormatividad social.

Celina cuenta que este proceso, que atravesaron en octubre de 2015, coincidió con la reciente reforma del Código Civil y tuvo lugar con la Ley de Matrimonio Igualitario plenamente vigente. La disputa de ambas madres se produjo ante la resistencia de los/as empleados/as de la institución a inscribir a la bebé como hija de ambas, argumentando que Celina, como madre no gestante, debía adoptarla. 
Este proceso implicó toda una movilización por parte de la pareja que da cuenta de lo que algunos/as autores/as denominan el parto social (Kaufman \& Morgan, 2005) y que en el relato de Celina surge de forma muy clara: "Yo realmente no la parí el día que nació, sino el día que fuimos al registro civil; cuando no me la quisieron inscribir y me dijeron que no era mi hija».

Frente a esta situación, Celina tuvo que recurrir a organizaciones de defensa de derechos LGBTQ y a los medios de comunicación, hasta que logró su reconocimiento como madre.

En el registro civil nos atendió una señora: «Sí, chicas, ¿qué andan buscando?» / «Acabamos de ser mamás y la venimos a inscribir» / «¿De ser mamás? ¿Cómo? ¿La adoptaste vos?» / «No, no. Las dos fuimos mamás porque hicimos un tratamiento, yo le transferí mis embriones a ella» / «iAh! Pero eso no se puede hacer. ¿Cómo que hiciste eso?» / «Sí, lo hizo un doctor en una clínica» / «iNo! Pero ustedes tiene que ir presas» / «No, señora, pero si yo vengo a inscribir a mi hija» / «Pero no es tu hija» / «No, es que sí, son mis embriones» / «Mirá, hoy día justo no nos andan las computadoras, así que hoy no se puede inscribir» / «Pero es que estamos acá hace un buen rato y estoy viendo que esa pareja que está ahí entró como si nada y están saliendo con el documento» / «No, no, porque ellos tenían otro papel que hacer» / «Pero si tienen un bebé chiquitito. Me lo estás diciendo porque ellos son hetero» / «iNo! Cómo va a ser por eso (...). Yo no sé qué es lo que han hecho ustedes, pero acá en la Argentina no está permitido eso, así que o la inscribe a nombre de ella y usted después le inicia un proceso de adopción...» / "iißPero cómo voy a adoptar a mi propia hija?! ¿Usted se da cuenta que ha cambiado el Código Civil? ¿Por qué no me explica qué tenemos que traerle para poder inscribir a mi hija?» (Celina, 43 años).

Este espacio de lo público como espacio político donde se dirimió el estatus de Celina como madre da cuenta, en primer lugar, de los sentidos heteronormativos y biologicistas presentes en las resistencias de los/as funcionarios/as que forman parte del Estado argentino. La alternativa de la adopción de su propia hija como propuesta inicial de estos/as agentes da cuenta de los límites de lo inteligible y de las resistencias que el mismo Estado presenta en determinados contextos sociales ante las transformaciones familiares y el reconocimiento de derechos. 
En segundo lugar, implica que el proceso de inscripción de los/as niños/as en entramados familiares de forma legal, algo que para la mayoría de las parejas supone un trámite rutinizado que pareciera refrendar un «hecho natural», constituye, en realidad, una decisión política sobre qué familias forman parte de las «familias naturales» y cuáles no.

En tercer lugar, permite visualizar que el parto social es un aspecto que forma parte de toda conformación familiar pero que en las familias disidentes a la heteronorma se elige invisibilizar. En la inscripción de los/as hijos/as de una pareja heterosexual no se piden pruebas de un vínculo biológico; sin embargo, para Celina esto se puso en discusión. Como señala Claudia Fonseca (2008), las familias homoparentales permiten poner en evidencia el papel que juegan el derecho, las tecnologías reproductivas y los recursos económicos en toda conformación familiar. Es decir, la maternidad de Celina implica una trayectoria con las TRHA, un conjunto de decisiones con su pareja y una disputa legal para lo cual fue central contar con otros recursos sociales y políticos que le permitieron lograr el reconocimiento de su familia. En este caso, implicó, además, disputar políticamente este reconocimiento partiendo de la propia identidad y experiencia reproductiva.

Si bien en la construcción de la maternidad de Celina fue muy importante que su hija llevara su material genético es, a la vez, el lugar social que ella construyó con su hija, con su pareja, con el personal médico, con las autoridades estatales, lo que define su papel como madre. Este aspecto, que aquí se hace explícito, no se presenta solo en el caso de las parejas de mujeres lesbianas, sino que da cuenta del proceso de construcción que toda mujer en posición de madre transita y performa.

\section{El original conyugal: madres solteras por elección}

La ruptura con la norma conyugal constituye otras de las dimensiones que se hace presente en los relatos de las usuarias, tanto desde el momento en el que deciden maternar ${ }^{10}$ sin una pareja, como durante y después del tratamiento. Si bien la monoparentalidad no es una novedad, las TRHA evidencian la elección, aspecto que sí es visto como un desafío a las normas sociales.

Más allá de la orientación sexual, la monoparentalidad elegida (Jociles Rubio \& Rivas Rivas, 2016) implica para las usuarias una ruptura con la heteronormatividad, debido a que es una elección que cuestiona el requisito de conyugalidad y de 
complementariedad en las figuras de cuidado, así como una disidencia frente a aquellas ideas vinculadas a la mirada biparental del cuidado y la crianza, propias de cierto modelo nuclear heterosexual.

En esta investigación se encontraron dos relatos de esta experiencia, contrastados por la edad de las entrevistadas. El de Candelaria, de 38 años, quien se encontraba en una relación con un varón y luego decidió ser madre soltera; y el de Hillary, de 49 años, quien se autoidentifica como lesbiana.

Candelaria se identificaba a sí misma como una candidata con pocas oportunidades para convertirse en adoptante, al entender que su condición de soltera no se correspondía con las representaciones hegemónicas de familia que espera el aparato estatal. En este sentido, ella considera que si bien el Estado no prohíbe la adopción por parte de una persona soltera prioriza a las parejas, mediante una lógica estratificada de lo familiar, como modelo ideal.

Es más fácil, yo lo veo con [nombre de su hija]. Es más fácil de lo que pensaba y es más intenso, y más difícil en otros puntos ¿no? Pero ese no es un impedimento, o sea, yo veo que en los trámites de adopción hacen mucho hincapié, mucho hincapié, en la cuestión económica... Y en la pareja, y yo no la tengo. Qué querés que haga, no tengo ganas de tener un ex, nunca buscar un novio, tener que buscar un ex, y después la familia, que tenga una familia piola, porque después es la familia a la que estás ligado de por vida... (Candelaria, 38 años).

$\mathrm{Su}$ relato pone en discusión el itinerario normativo para maternar, al disociar dos aspectos que la heteronorma familiar tiende a unir: la conyugalidad y el deseo de maternar como parte de un recorrido que legitima una decisión.

Perteneciente a una generación diferente a la de Canderalia, Hillary, de 49 años, relata que uno de los obstáculos que se le presentó al momento de decidir maternar sin pareja estuvo relacionado con cierto "habitus familiar incorporado»; al decir de Bourdieu (1997), con lo familiar normativo hecho cuerpo. 
Hay de las dos cosas, me parece, que tengo casi cincuenta años, o sea, he crecido. Cuando yo era chica, era muy raro que alguien tuviera los papás separados, ni siquiera existía el divorcio; entonces, yo he crecido con una figurita pegada a la cabeza de la estructura de familia convencional. Me parece que eso fue fuerte y fue parte de esta cosa de que estuviera más presente mientras yo no había definido más claramente mi sexualidad. Y me parece que esto de pensar en estar con una pareja para tener hijos todavía estaba presente, pero desde otro lugar también. Y también poder compartir con otro el tener hijos me parece que está bueno, está buenísimo. De hecho, en mi familia ha habido algunas de mis tías, que para esa época era rarísimo, que criaron a sus hijos solas, una cosa que (...) yo sé que se puede hacer (Hillary, 49 años).

Como señala al final del relato, si bien este habitus familiar está presente cuando se refiere a las dificultades iniciales para pensar su maternidad sin una pareja, al mismo tiempo, la experiencia familiar hecha cuerpo es recuperada cuando recuerda la experiencia de sus tías que maternaron sin una pareja, como ha sucedido históricamente, aun antes de la existencia de las TRHA.

Otro aspecto central de esta construcción de la monoparentalidad se vincula con ciertas transformaciones de sentidos a partir de las cuales la ausencia de una pareja no es sinónimo de soledad, así como tampoco la monoparentalidad es una situación sustituta de un ideal. Por el contrario, ambas usuarias dan cuenta de comunidades de cuidado que solo son posibles de pensar y de demandar cuando se rompe con la idea de que una pareja es garantía de un cuidado compartido, al tiempo que permite evidenciar que en toda crianza se precisa de una comunidad de socialización (Poveda, Rubio \& Rivas, 2011). Más aún, Candelaria y Hillary retoman los relatos de soledad de otras mujeres y madres en pareja con varones, donde lo que prima es la división sexual del trabajo de cuidado.

En su caso, Hillary señala que tuvo más acompañamiento que otras mujeres:

En un momento, conversando con una de mis primas que ha tenido cuatro hijos, me decía que veía que en esta situación en la que yo estaba sola había tenido mucha más ayuda, un acompañamiento que ella no había tenido de manera directa, aparte de su mamá. «Cuando tuve el primero, iba a la casa de mi mamá y ella me ayudaba con el bebé, pero esto que 
veo que pasa con vos se produce, justamente, porque estás sola... A veces se sobreentiende que la pareja se ocupa de todo». [Y no es tan así] No, a veces la pareja está totalmente desbordada... Hacen falta mucho más que dos personas, por lo menos durante primer mes, y justamente por esta situación o esta condición de estar sola, como diez, doce o quince personas estaban siempre atentas a qué hacía falta y colaboraban (Hillary, 49 años).

De esta manera, en ambos proyectos de maternidad la comunidad de lazos de cuidado que trasciende el parentesco es central para la conformación de sus familias.

[En referencia al discurso de una psicóloga de la clínica de TRHA] Me empezó a preguntar y me dijo: "Ah, pero vos no estás sola». Ahí yo medio como que me... «Vos no estás sola, vos tenés un equipo», porque yo le contaba que tenía a mis amigas, que estaban los tíos, que estaba toda mi familia, que estaba mi mamá, que había un montón de gente que la estaba esperando y que era un mundo muy diverso. Le dije: «Muy diverso. Yo me relaciono con gente de todo tipo (...) y quiero que mi hija viva ese mundo. La verdad, no necesito que me venga a completar, no, ni nada». Mi vida estaba tan buena que yo decía: «Debe ser re lindo ser hijo mío, vivir conmigo y que vamos juntas, va a estar bueno». A mí me pareció que iba a estar bueno (Candelaria, 38 años).

El relato de Candelaria no solo permite poner en discusión que la monoparentalidad elegida no constituye una experiencia estigmatizante, sino que es desde allí que ella construye su decisión y su deseo de maternar, lo que supone politizar desde la propia experiencia el original familiar.

Asimismo, estos relatos evidencian que más allá de la composición de las familias la relevancia de una comunidad de cuidado y de crianza transciende el requisito de la conyugalidad y supera la idea de un binomio parental. Como refiere Hillary, se vuelven necesarias «más de dos personas», por ello a su razonamiento se puede sumar un actor invisible pero central para una efectiva democratización de las condiciones de existencia de las familias: el Estado, que precisa ser un agente que democratice los cuidados para avanzar en una dirección que desfamiliarice esta tarea (Esquivel, Faur \& Jelin, 2012). 
Las experiencias de la adopción y la ovodonación en la conformación de las propias familias aparecieron, en algunos relatos de las usuarias, de forma comparada en el marco de su experiencia con las TRHA. Ello da cuenta de los diversos itinerarios y estrategias desplegados por las usuarias para acceder a la maternidad, en los que la adopción es una alternativa presente, en mayor o en menor medida, pero asimismo porque comparten una característica común: ambas constituyen experiencias que suponen algún tipo de pérdida de consanguinidad en la construcción familiar.

Entendiendo que la tecnología es siempre ideológica (Garay, 2008), desde las propias clínicas tiende a evitarse la ruptura con esta bioconexión, mediante la búsqueda de medios que se acerquen al modelo natural, lo que se expresa en la legislación vigente y en los protocolos. ${ }^{11}$ Para la donación de gametos, esto supone que en ocasiones se conserve la conexión genética por alguna línea -materna o paterna- a partir de priorizar el uso de gametos propios. La adopción aparece, también, como una de las alternativas para acceder a la maternidad y resulta, finalmente, en una vía exitosa para algunas entrevistadas como María y Sandra.

En los relatos, la ruptura con la maternidad natural que implican ambas elecciones se expresa en la comparación que realizan las usuarias, entendida como una experiencia que puede brindar elementos para otorgar inteligibilidad a la otra. El tránsito por prolongados tratamientos con TRHA es traído para pensar la decisión de adoptar como un proceso que les permitió tramitar subjetivamente la idea de la pérdida del lazo de sangre. Al mismo tiempo, encontramos relatos en los que el eje ordenador es la ovodonación, como elección que formó parte de la experiencia con las TRHA, y en los que la adopción como alternativa es puesta en consideración para pensar la inteligibilidad de la ovodonación. En palabras de una usuaria: «La ovodonación es como una adopción».

\section{La adopción como productora de inteligibilidad para la ovodonación}

La referencia a la adopción no supone en todos los relatos una opción posible y considerada por las usuarias, sino que aparece como una metáfora para pensar las posibilidades y las ventajas de la ovodonación frente a la adopción. Si bien en ambos casos se rompe con la consanguinidad, la posibilidad de gestación que permite el uso de ovodonación es valorada positivamente, como refleja el concepto 
de la uterización del vínculo (Viera Cherro, 2012), mediante el cual las usuarias valoran la posibilidad de ser gestantes aunque no sea con sus propios ovocitos. Así lo expresa Juliana, cuando refiere a su experiencia con ovodonación:

El médico me dijo, porque yo estaba así un poco que no entendía mucho, me dijo: «Va a ser tu hijo, vos lo vas llevar en tu vientre. Es tuyo». Y por el lado de la adopción, tenía mucho miedo a que me lo quiten. Sabía de muchas experiencias de gente que los había criado y después se los habían terminado sacando. Entonces, dije: «La verdad que nadie me lo va a venir a quitar». Bueno, eran como muchas cosas. Y ni hablar de la posibilidad de llevarlo en mi vientre, digamos, ni hablar (Juliana, 37 años).

De forma recurrente, la adopción aparece como una experiencia a comparar en la que se reflejan los temores de las mujeres usuarias frente a las TRHA y que les permitió a algunas usuarias tomar la decisión de utilizar ovodonación, al compararla con los procesos que supone la adopción. Tal es el caso de María, para quien la posibilidad de pensar en una adopción, cuando estaba realizando un tratamiento, le abrió camino a pensar sobre el uso de óvulos donados.

Que no iban a ser mis óvulos o que iban a hacer óvulos de otra persona con el esperma de mi esposo. Iba a ser hija de mi esposo, pero no hija mía. Esas fueron las primeras percepciones. Al principio, fue una cerrazón absoluta. Y después fue tiempo. Por eso fue largo todo ese estudio, fue decantando y empecé a pensar: «En definitiva, si nosotros estamos anotados para adopción tampoco iba a ser mía genéticamente, pero sí mía en el sentido del cariño de la relación conmigo». Y la psicóloga me ayudó a entender, porque me dijo: «Todo hijo es fruto de una adopción». Entonces, fue todo un camino de reflexión pensarlo (María, 43 años).

En el caso de Juliana, la alternativa de la adopción le permitió expandir las posibilidades reproductivas que parecían posibles en su repertorio decisional. De alguna manera, la adopción como una experiencia más familiar hace inteligible la práctica de la ovodonación, la cual resulta un proceso decisional que ubica a las 
usuarias como pioneras morales, como señala Rayna Rapp (1999), frente a prácticas que les resultan desconocidas, poco reguladas y que suponen un conjunto de decisiones muy complejas.

Asimismo, pone de manifiesto, al igual que en el caso de María -quien parte desde su experiencia de adopción- que «todo hijo es fruto de la adopción». Esta idea permite evidenciar que el proceso de maternaje y de crianza es un proceso afectivo, relacional, cultural que no se basa en ningún sustrato biológico, sino que hace referencia a todas las experiencias de cuidado, no solo a la adopción, aspectos que hacen posible cuestionar el modelo reproductivo estratificado.

\section{Las TRHA desde la experiencia de la adopción: el camino de ruptura}

La experiencia de María permite reflexionar sobre la manera en la que el itinerario terapéutico con las TRHA implica una transformación de sentidos a lo largo de ese trayecto, vinculado al cuestionamiento sobre la centralidad del valor de la conexión biológica y genética, aspecto que luego contribuyó a su decisión de adoptar. María cuenta cómo la experiencia de adoptar le permitió enfatizar que la maternidad se relaciona con un vínculo y con el afecto, y no con la gestación como condición.

Pienso, un poco a la fuerza y otro poco tomando conciencia, que los hijos no son solamente los que nacen de la sangre de uno, de la panza de uno... Son hijos del vínculo que uno crea, y del amor y el afecto que uno da. En ese sentido, a veces la veo a [nombre de su hija] y ni parece que no la hubiera tenido. No me acuerdo que no estuvo en la panza, se borró esa faceta de que tenerla en la panza significa que es tu hija. Y que si no son tus óvulos.... No son. Me parece que todo eso se cambió en una mentalidad mucho más abierta, y sí con mayor conciencia (María, 43 años).

Del mismo modo, supone un conjunto de pendientes frente a un original familiar. Así lo relata Sandra, quien registra las experiencias del embarazo y de la lactancia que algunas mujeres comparten como sinónimo de experiencia materna y de las cuales queda socialmente excluida. Lo que aquí está en juego no es la conexión genética sino la manera en la que el vínculo biológico -como el que se genera mediante la gestación y la lactancia- habilita en el proceso de cuidado y de crianza. 
A lo mejor te parece una tontera, pero es como una deuda pendiente que uno tiene siempre, esto de no haber logrado el embarazo. Es como una cosa pendiente, no pudiste sentir una vida adentro, no pudiste ver crecer la panza. Y tampoco podés intervenir en algunas conversaciones, cuando hablan del embarazo, del calostro y qué se yo... Lo sé todo porque escucho hablar a otros, pero no lo viví (Sandra, 44 años).

A su vez, relaciona esta deuda pendiente con una dimensión social, es decir, con las conversaciones y con ese saber que circula en los círculos de mujeres que comparten su experiencia de maternidad asociada a vivencias sobre el propio cuerpo que implican una conexión corporal que permite la gestación.

Hay una edad en que las mujeres se juntan a hablar de eso, de los hijos y del embarazo. "Ay, porque yo...». Y cuando empezaba el tema del embarazo, ya no tenía de qué hablar... Escuchaba para interiorizarme, pero siempre sentí que era una deuda pendiente que me quedó en la vida, ¿me entendés? Como tantas otras (Sandra, 44 años).

Estas exclusiones que experimentan aquellas madres por adopción, ponen de relieve que la conexión corporal se vuelve central para hacer inteligible la experiencia materna en determinados espacios sociales. Estas prácticas generan distancias que en la memoria biográfica de las usuarias construyen deudas sobra la experiencia materna, sustentadas en una noción de reproducción que da por sentada la conexión biológica o genética como experiencia reproductiva natural.

\section{Priorizar la familia original como camino posible}

En ocasiones, la adopción aparece en los relatos fuertemente imbricada en los procesos de estratificación reproductiva propios del contexto argentino. En el caso de Gisela, supone pensar el tratamiento tal como proponen los procedimientos clínicos y jurídicos, es decir, "agotando las vías naturales». Esto implica una 
progresión que parte del embarazo con gametos propios, pasa por la donación y los gametos de un solo progenitor, por los gametos donados y llega a la adopción como último recurso.

[En referencia a la adopción] Sí, en algún momento lo pensamos, pero decidimos que primero íbamos a agotar todos los caminos (no nos había resultado la de baja complejidad y fuimos a alta complejidad). Si no nos resultaba, es decir, hasta que no agotáramos todos esos caminos, recién ahí íbamos a ir (Gisela, 39 años).

Como relata Gisela, existe una lógica referida a «agotar todos los caminos» antes de adoptar, lo que se traduce en respetar la progresión que implica el tratamiento de las TRHA y el protocolo clínico. Pero en términos del parentesco, pasar de una técnica de baja complejidad a una de alta complejidad no solo supone la ruptura entre sexualidad y procreación, sino que en ocasiones también suma una ruptura con la conexión genética, como sucede cuando se utiliza donación de gametos, incluso aunque se conserve la conexión biológica por la posibilidad de gestar.

Otras formas en las que esto aparece en lo relatos se relaciona con la idea de «probar primero con el cuerpo», por la deseabilidad de un vínculo genético. En palabras de Celeste:

La verdad, está esto de lo biológico que lo tenemos como recontra incorporado. Pero nosotras como pareja siempre hablamos en relación a eso y ahora, más que nada, en relación a todo lo que tiene que ver con la crianza y los vínculos que se configuran más allá de lo genético... Entonces, creo que eso fue calmando un poco las aguas. Obviamente, eso es un duelo que tiene que atravesar ella y yo la puedo acompañar, pero es individual, es como muy, muy particular. Y sí, hemos tratado de recuperar -que es lo que siempre pensamos, no es que surge ahora- que la crianza es más importante que lo biológico. Siempre fue así, de hecho, en un momento estaba la posibilidad de pensar en una adopción (...) antes de pensar en tener un hijo más del propio cuerpo. No lo descartábamos, solo que dijimos: «Bueno, probemos con esto y en el caso que no suceda, porque tenemos 
varios intentos para probar, veremos con otra vía». Lo que pasa es que la otra vía sabemos que es bastante dolorosa, que lleva bastante tiempo, o sea, la adopción tampoco es tan fácil, entonces no fue la primera elección (Celeste, 36 años).

El relato de Celeste da cuenta de dos dimensiones relevantes en la decisión: por un lado, evidencia una ruptura con cierta construcción heteronormada de lo familiar; por otro, tampoco descarta el valor de esta conexión, cuando hace referencia al duelo por la pérdida de la consanguinidad. De esta manera, si bien Celeste no llega a inscribirse como adoptante, el uso de gametos donados y la adopción aparecen como dos prácticas tensionadas que permiten a las usuarias pensar sus decisiones.

Sin embargo, también da cuenta de un conjunto de representaciones sobre la adopción entendida como una alternativa burocrática e inaccesible. Esto lleva a pensar de qué manera el dispositivo biomédico, aunque en ocasiones doloroso, costoso y, en otros casos, ineficaz, es visualizado como una alternativa posible para quienes se identifican como disidentes de la heteronorma. Esta posibilidad da cuenta del sentido práctico (Bourdieu, 1992) al momento de tomar una decisión que impacta de forma más directa en las subjetividades que algunos reconocimientos jurídicos y sociales.

Este contraste entre ambas prácticas, tal como aparece en diversos relatos, se relaciona con las representaciones sociales sobre las TRHA como una vía más sencilla, más breve y menos disciplinante que la adopción, sobre la que existen representaciones sociales vinculadas al control social del Estado. Es así que estas prácticas aparecen nuevamente comparadas cuando se piensa en las complejidades que supone el tránsito por el dispositivo jurídico en la adopción, frente al dispositivo biomédico en la TRHA.

En este sentido, Celeste compara ambas prácticas, por considerar a las TRHA como una opción más simple:

Lo pensamos, sí, cuando dijimos: «Bueno, queremos empezar con este proyecto». Lo más sencillo era pensar en una fertilización, y en caso de que no resultara ninguno de los intentos que cubría la obra social -porque la verdad es que es un tratamiento bastante caro-. Si nada de eso resultaba, 
íbamos a pensar en alguna otra alternativa. (...) Creo que hay más cuestiones en contra que a favor de la adopción desde donde nosotros lo estamos vivenciando, sobre todo porque también implica... O sea, yo soy anti abogado, y por todo ese tema de papelerío la verdad es que prefiero inyectarme de vuelta, como fue el tratamiento este, antes que pasar de vuelta o atravesar todo ese malestar que sé que implica la adopción, por gente que lo ha pasado, ¿no? (Celeste, 36 años).

Similar señalamiento realiza Sofía, quien también consideró la adopción pero terminó desestimándola tras enfrentarse a un conjunto de obstáculos para hacerlo:

Me había hecho esa idea y tenía esta cosa como del prejuicio de que la fertilización asistida era un rebusque innecesario. Yo decía: "Qué necesidad con todos los chicos que hay para adoptar». Esa cosa de desinformación total... Porque ahora sé que adoptar es dificilísimo, y ahora tomé la decisión, decisiones que me fueron costando con respecto a esto de la fertilización asistida (Sofía, 44 años).

Estas reflexiones muestran que la comparación forma parte de las búsquedas, las decisiones y la información a la que acceden las usuarias de TRHA, para quienes la gestión del tiempo reproductivo supone optar por alguna vía, entre las cuales el dispositivo biomédico aparece como más fiable en comparación con el proceso jurídico de la adopción, idea asociada a una mayor accesibilidad y simplicidad frente a lo engorroso y complejo del dispositivo jurídico. En este orden de representaciones, el tratamiento reproductivo promete la gestión y el control del propio cuerpo, expectativa que no siempre es cumplida.

\section{Habilitar la disidencia frente al original familiar: resignificaciones y deudas pendientes}

El análisis de las categorías abordadas revela que existe una potencialidad en estas técnicas para desandar las prácticas normativas, entendiendo que las mismas han contribuido a evidenciar la creciente separación de la sexualidad y la reproducción 
en nuestra sociedad. Del mismo modo, las experiencias ponen de manifiesto que la disputa por la conformación y el reconocimiento de las propias familias permite poner en cuestión la idea de familia originada a raíz de una relación sexual heterosexual, conyugal y biológica como familia legítima. Esto llevaría a pesar que el uso de tales tecnologías ha contribuido a visibilizar otros sentidos de familia que han existido históricamente y que han sido estigmatizados.

Del mismo modo, ha permitido la politización de estas experiencias y la modificación de un sistema legal que no solo excluía a parejas de gays y lesbianas, sino a todo un entramado social vincular que se encontraba por fuera del binomio heterosexual nuclear. La realidad de las personas que ejercen el cuidado en diferentes arreglos familiares es un aspecto que ha sido históricamente invisibilizado en los diferentes programas y políticas sociales. La presencia o la ausencia de un vínculo genético o biológico es un aspecto que se toma como una referencia normativa de familia, pero que en la práctica nunca ha sido condición excluyente para los cuidados ejercidos por amiga/os, abuela/os, tías/os, educadoras/es y vecina/os. Asimismo, pone de relieve que más allá de la presencia o no de una pareja, la conformación de una comunidad de cuidados es una deuda del Estado hacia todos los sectores sociales.

Aquellas tensiones que aparecen en los relatos como culpas, pérdidas y dilemas, permiten acercarse desde la reflexividad de las propias experiencias a lo que es vivido como el costo que supone para las personas alejarse de este ideal normativo. Este costo no es solo subjetivo sino que se traduce en desigualdades -jurídicas, simbólicas, sociales- de las que son conscientes las usuarias de las TRHA. Se reconoce, entonces, que el campo de lo familiar reproduce las desigualdades y las jerarquías sociales, asentadas en una lógica heteropatriarcal. Ello supone que algunas conformaciones familiares obtienen mayores reconocimientos y privilegios en función de un original familiar. Quedar fuera de este reconocimiento social supone poner en riesgo los propios lazos y, en este sentido, la construcción de la identidad familiar debe ser disputada personal, social y hasta legalmente por algunas usuarias.

La disidencia frente al dispositivo heteronormativo, conyugal y bioconectado, implica diversas estrategias, recorridos y resignificaciones para quienes lo experimentan y posiciona a las usuarias frente a desafíos inesperados, que dan lugar a un conjunto de reflexiones sobre la construcción de lo familiar. 
Desde un sentido más amplio, estas tensiones suponen para los feminismos disputar el sentido de la definición familiar, no solo con el objeto de una mayor democratización de estos reconocimientos familiares, sino que apunte a cuestionar los fundamentos sobre los cuales se asientan las desigualdades en la reproducción social ampliada.

\section{Referencias}

Bourdieu, P. y Wacquant, L. (1992). An Invitation to Reflexive Sociology. Chicago, Estados Unidos: University of Chicago Press.

Bourdieu, P. (1997). Razones prácticas. Sobre la teoría de la acción. Barcelona, España: Anagrama.

Brettschneider, M. (2006). Family Flamboyant: Race Politics, Queer Families, Jewish Lives. Albany, United States: State University of New York Press,

Butler, J. (2002). Is Kinship Always Already Heterosexual? Differences, 13(1), 14-44. https://doi.org/10.1215/10407391-13-1-14

Colen, S. (1995). Like a Mother to Them. In F. Gynsburg y R. Rapp (Eds.), Conceiving the New Word Order. The Global Politics of Reproduction (pp. 78-102). Berkeley, Estados Unidos: University of California Press.

Conde, I. (1994). Falar da Vida (II). Revista Sociologia. Problemas e Práticas, (16).

Decreto 956 (2013). Ley Nacional 26.862. Reproducción Medicamente Asistida. Recuperado de http://servicios.infoleg.gob.ar/infolegInternet/anexos/215000- 
Esquivel, V., Faur, E. y Jelin, E. (Eds.) (2012). Las lógicas del cuidado infantil. Entre las familias, el Estado y el mercado. Ciudad Autónoma de Buenos Aires, Argentina: Instituto de Desarrollo Económico y Social (IDES).

Fonseca, C. (2008). Homoparentalidade: novas luzes sobre o parentesco. Revista Estudos Feministas, 16(3), 769-783. https://doi.org/10.1590/S0104-026X2008000300003

Garay, R. (2008). El destino de ser madres: la ideología de la maternidad como soporte discursivo de las nuevas tecnologías reproductivas. En O. M. Tarducci (Ed.), Maternidades en el siglo XXI (pp. 29-59). Ciudad Autónoma de Buenos Aires, Argentina: Espacio Editorial.

Hayden, C. P. (1995). Gender, Genetics and Generation: Reformulating Biology in Lesbian Kinship. Cultural Anthropology, 10(1), 41-63. https://doi.org/10.1002/9780470775981.ch7

Herrera, M. (2 de octubre de 2014). Principales cambios en las relaciones de familia en el nuevo Código Civil y Comercial de la Nación. Recuperado de http://www.saij.gob.ar/marisa-herreraprincipales-cambios-relaciones-familia-nuevo-codigo-civil-comercialnacion-dacf140723-2014-10-02/123456789-0abc-defg327041fcanirtcod

Jociles Rubio, M. I. y Villamil Pérez, F. (2012). Madres solteras por elección: representaciones sobre la fecundación sexual como vía de acceso a la maternidad. Chungará (Arica), 44(4), 717-731. https://doi.org/10.4067/S0717-73562012000400012

Kaufman, S. R. y Morgan, L. M. (2005). The Anthropology of the Beginnings and Ends of Life. Annual Review of Anthropology, (34), 317-341. https://doi.org/10.1146/annurev.anthro.34.081804.120452 
La Voz (26 de junio de 2013). Su pareja se embarazó, y Karina pide los 180 días por maternidad. Recuperado de https://www.lavoz.com.ar/ciudadanos/su-pareja-se-embarazo-karinapide-180-dias-maternidad

Ley de la Ciudad Autónoma de Buenos Aires 1.004 (2002).

Unión Civil. Recuperado de https://www.buenosaires.gob.ar/registrocivil/normativa/ley-no-1004

Ley Nacional 26.618 (2010). Matrimonio Civil. Recuperado de http://servicios.infoleg.gob.ar/infolegInternet/anexos/165000169999/169608/norma.htm

Ley Nacional 26.743 (2012). Identidad de Género. Recuperado de http://servicios.infoleg.gob.ar/infolegInternet/anexos/195000199999/197860/norma.htm

Ley Nacional 26.862 (2013). Reproducción Medicamente Asistida. Recuperado de http://servicios.infoleg.gob.ar/infolegInternet/anexos/215000219999/216700/norma.htm

Libson, M. C. (2012). Parentalidades gays y lesbianas: varones y mujeres en familias no heteronormativas. La ventana, 4(35), 292-321. https://doi.org/10.32870/lv.v4i35.731

Luna, N. (2001). Pessoa e parentesco nas novas tecnologias reprodutivas. Revista Estudos Feministas, 9(2), 389-413. https://doi.org/10.1590/S0104-026X2001000200005

Jociles Rubio, M. I. y Rivas Rivas, A. M. (2016). Cambios en la concepción y representación del parentesco a raíz del uso de las técnicas de reproducción asistida con donante. Ankulegi. Revista de Antropología Social, (20), 63-78. Recuperado de https://aldizkaria.ankulegi.org/index.php/ankulegi/article/view/93 
Peñas Defago, M. A., Moran Faundes, J. y Vaggione, J. M.

(6 de diciembre de 2018). Conservadurismos religiosos en el escenario global: amenazas y desafíos para los derechos LGBTI. Recuperado de https://sxpolitics.org/es/conservadurismos-religiosos-en-el-escenarioglobal-amenazas-y-desafios-para-los-derechos-lgbti/4167

Poveda, D., Rubio, M. I. J. y Rivas, A. M. (2011). Monoparentalidad por elección. Procesos de socialización de los hijos/as en un modelo familiar no convencional. Athenea Digital, 11(2), 133-154.

https://doi.org/10.5565/rev/athenead/v11n2.835

Preciado, B. P. (2019). Un apartamento en Urano. Crónicas del cruce. Barcelona, España: Anagrama.

Rapp, R. (1999). Testing Women, Testing the Fetus: The Social Impact of Amniocentesis in America (Anthropology of Everyday Life). New York, United States: Routledge.

Rapp, R. (2001). Gender, Body, Biomedicine: How Some Feminist Concerns Dragged Reproduction to the Center of Social Theory. Medical Anthropology Quarterly, 15(4), 466-477. https://doi.org/10.1525/maq.2001.15.4.466

Rose, N. (2007). The Politics of Life it Self. Biomedicine, Power and Subjetivity in the Twenty First Century. Princeton, New Jersey: Princeton University Press.

Sautu, R., Boniolo, P., Dalle, P. y Elbert, P. (2005). Manual de metodología. Construcción del marco teórico, formulación de los objetivos y elección de la metodología. Ciudad Autónoma de Buenos Aires, Argentina: Consejo Latinoamericano de Ciencias Sociales (CLACSO). 
Sgró Ruata, M. C. (2012). ¡Queremos Mamá y Papá! Cruces políticoreligiosos en la oposición al matrimonio igualitario en Córdoba (Argentina, 2010). Revista de Antropología y Sociología de la Universidad de Caldas, 14(2), 129-156. Recuperado de http://vip.ucaldas.edu.co/virajes/downloads/Virajes14(2)_8.pdf

Strathern, M. (1992). Reproducing the Future. Antropology, Kinship, New Reproductive Tecnologies. Manchester, England: Manchester University Press.

Strathern, M. (1995). Necessidade De Pais, Necessidade De Mães. Estudos Feministas, (2), 303-329.

Thompson, C. (2005). Making Parents. The Ontological Choreography of Reproductive Technologies. Cambridge, England: MIT Press books.

Vaggione, J. M. (2008). Las familias más allá de la heteronormatividad. En C. Motta y M. Sáez (Eds.), La mirada de los jueces, Tomo II (pp. 13-88). Bogotá, Colombia: Siglo del Hombre.

Vaggione, J. M. (2012). Introducción. En Morán Faúndes, J. M., Sgró Ruata, M. C. y Vaggione, J. M. (Eds.), Sexualidades, desigualdades y derechos. Reflexiones en torno a los derechos sexuales $y$ reproductivos (pp. 13-55). Córdoba, Argentina: Editorial de la Universidad Nacional de Córdoba.

Vespucci, G. (2014). Una fórmula deseable: el discurso «somos familias» como símbolo hegemónico de las reivindicaciones gaylésbicas. Sexualidad, Salud y Sociedad (Rio de Janeiro), (17), 30-65. https://doi.org/10.1590/1984-6487.sess.2014.17.05.a

Viera Cherro, M. (2012). Inequidades múltiples y persistentes en el campo de la reproducción asistida. Revista de Antropología Social, (21), 251-271. https://doi.org/10.5209/rev_RASO.2012.v21.40058 


\section{Notas}

1 «Contemporary infertility and its treatment are conceptualized and structured around a strongly coupled, heterosexual, consumeroriented, normative nuclear-family scenography. When successful, treatment enables women to reinscribe themselves into that logic» (Thompson, 2005, p. 56). Traducción de la autora del artículo.

2 Se utilizará la noción "familia bioconectada» para hacer referencia a lo que se denomina como «lazos de sangre» o consanguinidad. Su uso no desconoce la diversidad de situaciones producto de las TRHA; en ocasiones, el uso de donantes de gametos posibilita una "conexión biológica» por la posibilidad de gestación pero no una «conexión genética».

3 Tesis titulada: Nuevas tecnologías reproductivas: sentidos sobre el parentesco en las trayectorias biográficas de mujeres de Córdoba. Universidad Nacional de Córdoba. Facultad de Ciencias Sociales. Centro de Estudios Avanzados. Doctorado en Estudios de Género. Defendida en marzo de 2019. Dicha tesis fue financiada con una beca doctoral otorgada por el Consejo Nacional de Investigaciones Científicas y Técnicas (CONICET).

4 Se hará referencia a mujeres, pero es preciso señalar que las personas entrevistadas son reconocidas como cis mujeres, es decir, mujeres en las que coincide su identidad de género con el sexo asignado al nacer. Si bien la técnica de bola de nieve permitió lograr una muestra diversa respecto a la edad, el momento del tratamiento, la orientación sexual y la situación conyugal, fue homogénea en el aspecto educativo y en la identidad de género de las usuarias, siendo estos aspectos una limitación en el estudio.

5 De acuerdo con las clasificaciones de las sociedades de medicina reproductiva, se puede distinguir entre técnicas de baja complejidad como la inseminación artificial (con o sin donante de esperma) y técnicas de alta complejidad como la fecundación in vitro (FIV) y la inyección intracitoplasmática de espermatozoide (ICSI), utilizando gametas propias (homologas) o gametas donadas, que en los casos recabados fueron con ovodonación.

6 A partir de un acuerdo de confidencialidad en el tratamiento de los datos, los nombres de las personas entrevistas en esta investigación han sido resguardados.

7 El Programa Médico Obligatorio (PMO) es una canasta básica de prestaciones obligatorias que toda obra social o prepaga debe cubrir como mínimo en cualquiera de sus planes.

8 «[...] we must see that the natural family is itself not natural. [...] We will find that the situation is not as simple as: the biological family is given, while the adoptive family is made; the biological family is natural, while the adoptive family is constructed; or that the biofamily is real or original and the adoptive family a copy or mirror. In contrast, I will show that, under scrutiny, the framework "adoptive families are to culture as bio-families are to nature" does not hold» (Brettschneider, 2006, p. 81). Traducción de la autora del artículo. 
9 Lo mismo sucedió en Córdoba, en 2013, con otra pareja de mujeres, cuando la madre no gestante solicitó licencia por maternidad. Luego de entrar en una contienda con la policía de la Provincia de Córdoba, donde trabajaba, fue despedida y vulnerada en sus derechos (La Voz, 26/06/2013).

10 Se hace referencia a la decisión de "maternar», y no a la decisión de "ser madre», porque como surgió en el marco de esta investigación las usuarias no se refieren a la maternidad como una característica identitaria, sino como una construcción asociada a una práctica de cuidado.

11 El Programa Nacional de Reproducción Médicamente Asistida (creado en 2016 a partir de la reglamentación de la Ley 26.862/13 de Reproducción Medicamente Asistida) señala, entre otros aspectos, el respeto de una gradualidad en el uso de las TRHA que tiene su correlato en los costos. De esta manera, es esperable que una usuaria que comience con los tratamientos inicie el recorrido con la inseminación artificial (la técnica más simple y accesible), mientras que hacerlo desde una técnica de mayor complejidad -como la FIV o la ICSI- queda sujeto a criterios médicos que así lo justifiquen (Decreto 956/13, art. 8). 